Original Research Paper

\title{
Glucans and the Poultry Immune System
}

\author{
Jacqueline Jacob and Anthony Pescatore \\ Department of Animal and Food Sciences, University of Kentucky, KY, USA
}

\author{
Article history \\ Received: 15-11-2016 \\ Revised: 10-01-2017 \\ Accepted: 29-03-2017 \\ Corresponding Author: \\ Jacqueline Jacob \\ Department of Animal and \\ Food Sciences, University of \\ Kentucky, KY, USA \\ Tell: 859-257-7613 \\ Email: Jacquie.jacob@uky.edu
}

\begin{abstract}
With the reduced availability of antibiotics, poultry producers are looking for feed additives to stimulate the immune system of their birds. Some beta-glucans have been shown to improve gut health in poultry subjected to a bacterial challenge, to increase the flow of new immunocytes into the various lymphoid organs, to increase macrophage function, to increase antibody titers after a vaccination and to function as an anti-inflammatory immunomodulator. As a result, beta-glucans may provide a tool for producers trying to reduce or eliminate the use of antibiotics in poultry diets.
\end{abstract}

Keywords: Glucans, Poultry, Immunity

\section{Introduction}

Bacterial infections in poultry are an important concern for both animal health and productivity. In addition, some bacteria are food borne pathogens like Salmonella and Campylobacter. The subtherapeutic use of antibiotics has reduced the impact of bacterial infections, but has led to concerns regarding development of antibiotic resistance. The result has been the withdrawal of several antibiotics from the toolbox available to poultry producers. As poultry producers work to reduce the use of antimicrobials, alternative management strategies are being developed. There has been increased interest in 'natural' feed additives that can stimulate the immune system of poultry. There has been some research to show that $\beta$-glucans may play a role in replacing antibiotics and stimulating the immune system. Rathgeber et al. (2008) reported that yeast $\beta$-glucan was as effective in promoting growth of broiler chickens as virginiamycin, an antibiotic routinely added to broiler diets at subtherapeutic levels. Similarly, Zhang et al. (2008) reported that supplementing broiler diets with $\beta$-glucans can improve growth performance in terms of both average daily gain and feed conversion ratio.

The immunomodulatory effects of $\beta$-glucans have been well documented in mammals, but, while the mammalian and avian immune systems are similar, they are different enough that it is not possible to extrapolate mammal research to poultry.

\section{Glucans}

Glucans are complex glucose polymers that are structural components of the cell walls of many organisms including bacteria, yeast, fungi and algae. They are also found in the cereals oats and barley. The six-sided glucose rings are connected together in linear or branched forms with glyosidic linkages. The nature of these linkages will affect the functionality of the molecules. The glucans have a backbone of glucose molecules linked at the 1 and 3 carbon atoms. Bacterial glucans are simply this backbone of glucose molecules. Fungal and yeast glucans, however, have side-chains linked at the 1 and 6 carbon atoms. This gives them a branched structure. Comparing the two, fungal glucans typically have shorter side chains than those found in yeast. By comparison, barley glucans have the glucose molecules linked at the 1 and 4 carbon atoms with some linkages between the 1 and 3 carbon atoms. The $1 \rightarrow 3$ linkages give the molecule a semi-flexible nature and the $1 \rightarrow 4$ add kinks to that chain.

Table 1. Types of $\beta$-glucan linkages generally found in different sources

\begin{tabular}{ll}
\hline Source & Linkage \\
\hline Bacteria & $(1 \rightarrow 3)$ \\
Fungal & $(1 \rightarrow 3)(1 \rightarrow 6)$ with \\
& short branches \\
Yeast & $(1 \rightarrow 3)(1 \rightarrow 6)$ with \\
& longer branches \\
Cereal (barley and oats) & $(1 \rightarrow 3)(1 \rightarrow 4)$ \\
\hline
\end{tabular}




\section{Effect of $\beta$-Glucan Supplementation on Poultry Immune System}

The intestinal lining provides the innate defense barrier against most intestinal pathogens. A Salmonella Thyphimurium challenge has been shown to have major negative effects on the intestinal mucosa (Shao et al., 2013). There is a significant reduction in villus height and the villus height to crypt depth ratio in the jejunum. Dietary yeast $\beta$-glucan supplementation for chicks challenged with $S$. Thyphimurium, however, resulted in higher villus height and villus height to crypt depth ratio than for the unsupplemented chicks (Shao et al., 2013). Goblet cells in the intestinal mucosa secrete mucus which provides the first line of defense against intestinal injury. In $S$. Thyphimurium challenged chicks, the number of goblet cells decreased. Supplementation with $\beta$-glucan, however, significantly increased the number of goblet cells in the jejunum of challenged chicks (Shao et al., 2013). This suggests that the $\beta$-glucan is playing a role in improving gut health during a bacterial challenge.

All cells involved in the immune reactions originate from common precursors. $\beta$-glucan stimulates the production of precursor cells in bone marrow, resulting in an increase flow of new immunocytes into the various lymphoid organs throughout the body (Petravić-Tomenac et al., 2010). This increases protection from potential invaders.

Macrophages are large white blood cells that phagocytize invading pathogens (Qureshi, 2003). In a typical immune response, macrophages recognize specific markers on the surface of the pathogens, referred to as Microbial Associated Marker Proteins (MAMPs). The MAMPs bind to the receptors on the phagocytic cells known as Toll-like Receptors (TLRs) (Medzhitov et al., 1997). Birds have 10 known TLRs, although only five are orthologous to both birds and mammals (Temperley et al., 2008). Key TLRs in chickens are TLR-2 that recognizes peptidoglycan, TLR4 that binds LPS common in gram-negative bacteria, TLR-5 that recognizes flagellin common in bacteria flagella and TLR-21 which recognizes unmethylated CpG DNA commonly found in bacteria (Keestra et al., 2010). The macrophage binds the MAMPs of the invading pathogens to the TLRs on their outer surface and brings the organism into their cytoplasm in the form of a phagosome. The phagosome then fuses with a lysosome and the enzymes in the lysosome degrade the engulfed organism. Macrophage function has been shown to be responsive to dietary $\beta$-glucans. Dietary supplementation with $\beta$-glucans from the yeast Saccharomyces cerevisiae has been shown to increase phagocytic activity in broiler chicks (Guo et al., 2003). Lowry et al. (2005) reported that feeding day-old chicks with purified $\beta$-glucan significantly decreased the levels of Salmonella Enteritidis (SE), a potential human pathogen, in the liver and spleen. The $\beta$-glucans appear to have functioned by increasing phagocytosis, bactericidal killing and oxidative burst from the heterophils. This was confirmed by Chen et al. (2008) who reported that supplementation with yeast $\beta$-glucan enhanced the chick's defense against SE by directly upregulating both the phagocytosis and bactericidal activity of abdominal macrophages. Dietary supplementation of $\beta-1,3-1,6$-glucan of the fungus Schizophyllum commune was also shown to increase the phagocytic and bactericidal capabilities of intestinal macrophages (Chen et al., 2008; Muthusamy et al., 2013).

Macrophages also produce the enzyme iNOS. This enzyme results in the production of nitric oxide which reacts with superoxide anions to generate toxic derivatives which allow the macrophage to kill several types of pathogens. Cox et al. (2010) reported upregulation of iNOS in response to $\beta$-glucan supplementation, implying increased capacity of macrophages to destroy pathogens.

Animals are able to recognize cells as being self from those that are not self. This is done through specific markers on the surface of the body cells referred to as Major Histocompatibility Complex (MHC). The TLRs are non-self-proteins on invading organisms and are referred to as antigens because they stimulate the production of antibodies. When a macrophage digests a pathogen it does not destroy the antigens. Instead, the antigens are processed and moved to the surface of the macrophage producing a MHC-antigen complex and creating an antigen-presenting macrophage which triggers the adaptive immune system.

As with mammals, the avian adaptive immune system has two major segments: Humoral and cellular immunity. Humoral immunity involves antibody production from B-lymphocytes which are associated with the Bursa of Fabricius, which is unique to birds. The principal cells involved in cellular immunity are Tlymphocytes which are associated with the thymus. After the foreign cell is degraded, the macrophage presents bacterial peptides or antigenic determinants to T- and Bcells (Qureshi, 2003; Genovese et al., 2013).

There are different types of T-cells. The cytotoxic Tcell (Tc) binds to the MHC-antigen complex on the macrophage. The macrophage then secretes the chemical Interleukin-1 (IL-1). In response, the type-1 helper T-cell (Th1) binds to another MHC-antigen on the macrophage and releases Interleukin-2 (IL-2). IL-2 then activates the already bound Tc, causing it to go through several rounds of cell division to create a large population of Tc cells. The population of Tc cells then divides into two subpopulations of effector cells, which will go out and destroy infected body cells and memory cells, which are 
held in reserve for future exposures to the pathogen. The effector cells will bind to infected body cells and secrete perforins which punch holes in the surface of the infected cell causing it to spill out its contents. The effector cell then moves on in search of other infected body cells. After the infection is over, the number of effector cells will decrease.

Macrophages also produce IL-1 $\beta$, interferon (IFN)- $\gamma$, IL-2, Tumor Necrosis Factor (TNF)- $\alpha$ and IL-4. The production of IL- 4 results in the development of type- 2 helper T Cells (Th2). Th2 cells than produce IL-5, IL-6, IL-10 and IL-13 which are involved in the humoral immune response. Macrophages can also produce IL-8 (sometimes referred to as CXCLi2), which is a chemokine that is an important mediator of the innate immune system. Its primary function is to induce the migration of its target cells to inflammation sites (Cox et al., 2010). IL-18 is a pro-inflammatory cytokine largely produced by macrophages. After exposure to a pathogen, IL-18 works with IL-12 to induce a cell-mediated immune response. It primarily targets Th cells which will then secrete interferon- $\gamma$. IFN- $\gamma$ plays an essential role in activating macrophages (Cox et al., 2010).

$\beta$-glucan supplementation has been shown to alter the cytokine profiles of unchallenged, day-old chicks (Cox et al., 2010). Compared with unsupplemented controls, broiler chicks receiving $\beta$-glucan from Saccharomyces cerevisiae were shown to down-regulate IL-8. The down-regulation of the IL- 8 gene with $\beta$ glucan supplementation suggests that the $\beta$-glucan functions as an anti-inflammatory immunomodulatory (Cox et al., 2010).

Cox et al. (2010) were the first to report on the effects of dietary $\beta$-glucan on IL-18. Expression of the pro-inflammatory cytokine IL-18 was found to be upregulated in the jejunum seven days after feeding $\beta$ glucan to day-old chicks. By day 14, however, there was a decrease in IL-18 in the duodenum. IFN- $\mathrm{Y}$, which plays a central role in promoting Th1 cell differentiation and activating macrophages, was reported to be downregulated in the $\beta$-glucan supplemented chicks compared to those receiving the unsupplemented control diet. Supplementation with $\beta$-glucan for seven-days posthatch up-regulated IL-4 expression in the duodenum, jejunum and ileum. By day 14, however, IL-4 expression was up-regulated in the duodenum and ileum (Cox et al., 2010). The functions of IL-13 overlap with those of IL4. Similar to what happened with the IL-4 expression on day seven, IL-13 gene expression was also downregulated in all intestinal sections. Having both IL-4 and IL-13 being down-regulated would suggest that $\beta$-glucan supplementation does not support a Th2 cell-mediated response (Cox et al., 2010).

It has been hypothesized that $\beta$-glucans link to the Major Histocompatibility Complex (MHC) and subsequently activate monocytes and macrophages (Zhang et al., 2008). This results in increased immune response including the activation of cytotoxic macrophages, T-helper and natural killer cells, as well as the promotion of $\mathrm{T}$-cell proliferation and differentiation. IL-1, IL-2, IFN- $\gamma$ and TNF- $\alpha$ may act as the signal regulating this immune network.

B-cells are lymphocytes with bound antibodies on their surface. The antibodies will match specific antigens typically found on pathogens. When the B-cell with the antibody for a specific antigen encounters the pathogen with this antigen, the two become bound together, priming the B-cell. The IL-2 from the T-cell acts as a signal for the B-cell to divide to create a large population of B-cells. The B-cells divide into two subpopulations, effector and memory cells. The effector cells are involved in the production of antibodies while the memory cells remain in preparation for future infections. The antibodies will circulate in the blood and attach to the pathogen. It is difficult for the pathogen to infect other body cells when the antibodies are attached. The antibodies also mark the pathogens for destruction. The destruction can be carried out by macrophages, natural killer cells and heterophils. Natural Killer (NK) cells are a type of cytotoxic lymphocyte. They play a role similar to cytotoxic T-cells. Heterophils are granulocytic phagocytes and functionally equivalent to neutrophils in mammals. The cytoplasmic granules of heterophils contain lysozyme and proteins needed for bactericidal activity. Unlike mammalian neutrophils, however, heterophils do not have peroxylase activity. Inclusion of yeast $\beta$-glucan and a commercial probiotic product in the diet of chicks resulted in significantly higher antibody titers against Newcastle disease or infectious bronchitis virus as compared to unsupplemented control chicks (An et al., 2008).

The intestinal tract is an important interface between the external environment and the internal body itself. Gut immunity, therefore, is important as the first line of defense from various pathogenic organisms. In the chicken, the Gut-Associated Lymphoid Tissues (GALT) include cecal tonsils and Peyer's patches, as well as lymphoid aggregates in the urodeum and proctodium of the cloaca. GALT structures are the sites of immune induction including the production of Immunoglobulin A (IgA) from B-cells. IgA is found in serum bile, mucosal surfaces, saliva and tears. They function in mucosal immunity. They neutralize virus and microbial toxins and prevent the adherence and colonization of microbial pathogens. As such, IgA is an important intestinal defense. A $S$. Thyphimurium challenge increased the number of IgA-expressing cells as well as the level of IgA produced. These levels were even higher in the chickens receiving $\beta$-glucan supplemented diets (Shao et al., 2013). $\beta$-Glucan supplementation also reduced the levels of $S$. 
Thyphimurium infiltration as indicated by lower cecal and liver levels.

Much of the research with $\beta$-glucans and poultry has been with yeast products. Cheng et al. (2004) showed that $\beta$-1,3-glucan of the fungus Schizophyllum commune significantly increased the chemotaxis activity of macrophages, but had no effect on lymphocyte blastogenesis after exposure to different mitogens and no effect on antibody titer after a Newcastle Disease vaccination. The difference in results may be the level of $\beta$-glucan used since Cheng et al. (2004) used low levels of $\beta$-glucan inclusion.

A $\beta$-glucan from mushrooms has been shown to promote splenocyte proliferation and interleukin-2 production in broilers (Chen et al., 2008). Purified $\beta$ glucan from the edible mushroom Pleuratusflorida was shown to provide broiler chicks with some protection from Newcastle disease (Paul et al., 2012). IL-2 production was reported to have increased significantly.

Zhang et al. (2012) fed a commercial $\beta$-glucan powder to broilers. The $\beta$-glucan was derived from the bacterium Agrobacterium species. The powder was guaranteed to contain $86.1 \% \beta-1,3 / 1,6$ glucan. While Zhang et al. (2012) did not observe any changes in blood profiles, feeding $\beta$-glucan significantly increased (3.9\%) the relative weight of the spleen. In chickens the spleen serves as both a reservoir of leukocytes as well as their activation. Activation of spleen genes reflects systemic immune function (Redmond et al., 2010). This would include genes responsible for interleukin (IL)-4 and IL18. Different chicken genotypes have been shown to react differently to $\beta$-glucan supplementation (Redmond et al., 2010). Gene expression of IL-4 and L18 in a broiler line was not changed in response to a 3week $\beta$-glucan supplementation, but did respond in chickens of the Fayoumi line. This would indicate that the effect of immunomodulatory supplementation may not have the same beneficial effects in all gene lines.

An additional impact of $\beta$-glucans on the immune system involves protection from mycotoxins. Mycotoxins such as aflatoxin B1 (AFB1) cause major health issues in poultry. Chronic AFB1 intake has been shown to result in poor health, a loss in productivity, as well as immune system dysfunction. This appears to be due to the damage to cell DNA (Zimmermann et al., 2014). $\beta$-Glucans from the yeast Saccharomyces cerevisiae have been shown in vitro to be cytoprotective and genoprotective of chicken lymphocytes (Zimmermann et al., 2015). The researchers speculated that the cytoprotection was a result of $\beta$-glucan assisting in repair or blocking of mutations in the life cycle of the cell. This could be due to $\beta$-glucan adsorbent capacity, interacting chemically with AFB1.

Le et al. (2011) reported that a commercial $\beta$-glucan was a potential effector, enhancing the immune response of the avian influenza $\mathrm{H} 5$ vaccines tested. The avian influenza vaccines produced in oil-emulsified inactivated form, were shown to induce immunity in chickens, Muscovy ducks and Vietnamese ducks. They added a $\beta$ 1,3-1,6 glucan produced by the black yeast Aureobasidium pollulans to the vaccine and assessed the ELISA and hemagglutination inhibition assays. Animals with the $\beta$-glucan enhanced vaccine had titers significantly higher than those vaccinated with the unsupplemented vaccine.

\section{Conclusion}

There are different types of $\beta$-glucans. Glucans from cereals such as barley and oats, have very detrimental effects on poultry health and performance. The inclusion of some of the varieties of barley and oats in poultry diets is made possible by supplementation with feed enzymes which digest the $\beta$-glucan. Other $\beta$-glucans, such as those from fungi and yeast, have been shown to be beneficial as dietary supplements. They have been shown to increase immune responses in poultry. $\beta$-Glucan supplementation was shown to increase the production of new immunocytes. Supplementation has also been shown to increase the phagocytic function of macrophages. $\beta$ Glucans increase the phagocytic, bactericidal killing and oxidative burst of heterophils. $\beta$-Glucan has also been shown to enhance immune response by altering the cytokine profiles of chickens.

$\beta$-Glucans, therefore, may provide a tool for producers trying to reduce or eliminate the use of antibiotics in poultry diets. Supplementation with $\beta$-glucans has also been shown to be beneficial in protection from mycotoxins. In addition, inclusion of $\beta$-glucans in vaccines, may enhance the immune response they elicit.

\section{Author's Contributions}

Both authors contributed to the writing of this paper.

\section{Ethics}

No ethical issues are expected to arise from the publication of this manuscript.

\section{References}

An, B.K., B.L. Cho, S.J. You, H.D. Paik and H.I. Chang et al., 2008. Growth performance and antibody response of broiler chicks fed yeast derived $\beta$-glucan and single-strain probiotics. Asian-Aust. J. Anim. Sci., 21: 1027-1032. DOI: 10.5713/ajas.2008.70571

Chen, K.L., B.C. Weng, M.T. Chang, Y.H. Liao and T.T. Chen et al., 2008. Direct enhancement of the phagocytic and bactericidal capability of abdominal macrophage of chicks by $\beta-1,3-1,6$-glucan. Poult. Sci., 87: 2242-2249. DOI: 10.3382/ps.2008-00147 
Cheng, Y.H., D.N. Lee, C.M. Wen and C.F. Weng, 2004. Effects of $\beta$-glucan supplementation on lymphocyte proliferation, macrophage chemotaxis and specific immune responses in broilers. Asian Aust. J. Anim. Sci., 17: 1145-1149. DOI: 10.5713/ajas.2004.1145

Cox, C.M., L.H. Stuard, S. Kim, A.P. McElroy and M.R. Bedford et al., 2010. Performance and immune responses to dietary $\beta$-glucan in broiler chicks. Poult. Sci., 89: 1924-1933. DOI: 10.3382/ps.2010-00865

Genovese, K.J., H. He, C.L. Swaggerty and M.H. Kogut, 2013. The avian heterophil. Dev. Comparative Immunol. 41: 334-340. DOI: $10.1016 /$ j.dci.2013.03.021

Guo, Y., R.A. Ali and M.A. Qureshi, 2003. The influence of $\beta$-glucan on immune responses in broiler chicks. Immunopharmacol. Immunotoxicol., 3: 461-472. DOI: 10.1081/IPH-120024513

Keestra, A.M., M.R. de Zoete, L.I. Bouwman and J.P. van Putten, 2010. Chicken TLR21 is an innate CpG DNA receptor distinct from mammalian TLR9. J. Immunol., 185: 460-467. DOI: 10.4049/jimmunol.0901921

Le, T.H., T.B. Le, T.H.T. Doan, D.V. Quyen and K.X.T. Le et al., 2011. The adjuvant effect of Sophy $\beta$-glucan to the antibody response in poultry immunized by the avian influenza A H5N1 and H5N2 vaccines. M. Microbiol. Biotechnol., 21: 405-411. PMID: 21532325

Lowry, V.K., M.B. Farnell, P.J. Ferro, C.L. Swaggerty and A. Bahl et al., 2005. Purified $\beta$-glucan as an abiotic feed additive up-regulates the innate immune response in immature chickens against Salmonella enterica serovar Enteritidis. Int. J. Food Microbiol., 98: 309-318.

DOI: $10.1016 /$ j.ijfoodmicro.2004.06.008

Medzhitov, R., P. Preston-Hurlburt and C.A. Janeway Jr., 1997. A human homologue of the Drosophila Toll protein signals activation of adaptive immunity. Nature, 388: 394-397. DOI: 10.1038/41131

Muthusamy, G., S.N. Joardar, I. Samanta, D.P. Isore and B. Roy et al., 2013. $\beta$-Glucan from edible mushroom (Pleurotus florida) enhances mucosal immunity in poultry. Adv. Anim. Vet. Sci., 1: 116-119.

Paul, I., D.P. Isore, S.N. Joardar, I. Samanta and U. Biswas et al., 2012. Orally administered $\beta$-glucan of edible mushroom (Pleuratusflorida) origin upregulates innate immune response in broilers. Indian J. Anim. Sci., 82:745-748.

Petravić-Tomenac, V., V. Zechner-Krpan, S. Grba, S. Screčec and I. Panjkota-Krbavčić et al., 2010. Biological effects of yeast $\beta$-glucan. Agric. Conspectus Scientificus, 75: 149-158.

Qureshi, M.A., 2003. Avian macrophage and immune response: An overview. Poult. Sci., 82: 691-698. DOI: $10.1093 / \mathrm{ps} / 82.5 .691$
Rathgeber, B.M., K.L. Budgell, J.L. MacIsaac, M.A. Mirza and K.L. Doncaster, 2008. Growth performance and spleen and bursa weight of broilers fed yeast betaglucan. Can. J. Anim. Sci., 88: 469-473.

DOI: $10.4141 /$ CJAS07101

Redmond, S.B., R.M. Tell, D. Coble, C. Mueller and D. Palić et al., 2010. Differential splenic cytokine responses to dietary immune modulation by diverse chicken lines. Poult. Sci., 89: 1635-1641. DOI: $10.3382 /$ ps.2010-00846

Shao, Y., Y. Guo and Z. Wang, 2013. $\beta-1,3 / 1,6-G l u c a n$ alleviated intestinal mucosal barrier impairment of broiler chickens challenged with Salmonellaenterica serovar Typhimurium. Poult. Sci., 92: 1764-1773. DOI: $10.3382 /$ ps.2013-03029

Temperley, N.D., S. Berlin, I.R. Paton, D.K. Griffin and D.W. Burt, 2008. Evolution of the chicken toll-like receptor gene family: A story of gene gain and gene loss. BMC Genom., 9: 62-74. DOI: $10.1186 / 1471-2164-9-62$

Zhang, B.Z., Y. Guo and Z. Wang, 2008. The modulating effect of $\beta-1,3 / 1,6$-glucan supplementation in the diet on performance and immunological responses of broiler chickens. AsianAust. J. Anim. Sci., 21: 237-244. DOI: 10.5713 ajas.2008.70207

Zhang, Z.F., T.X. Zhou, X. Ao and I.H. Kim, 2012. Effects of $\beta$-glucan and Bacillus subtilis on growth performance, blood profiles, relative organ weight and meat quality in broilers fed maize-soybean meal based diets. Livestock Sci., 150: 419-424. DOI: 10.1016/j.livsci.2012.10.003

Zimmermann, C.E.P., A.K. Machado, F.C. Cadoná, J.A.S. Jaques and K.B. Schlemmer et al., 2014. Invitro cytotoxicity of aflatoxin $\mathrm{B} 1$ to broiler lymphocytes of broiler chickens. Braz. J. Poultry Sci., 16: 307-312.

DOI: $10.1590 / 1516-635 \times 1603307-312$

Zimmermann, C.E.P., I.B.M. Cruz, F.C. Cadoná, A.K. Machado and C. Assmann et al., 2015. Cytoprotective and genoprotective effects of $\beta$ glucans against aflatoxin $\mathrm{B}_{1}$-induced DNA damage in broiler chicken lymphocytes. Toxicol. Vitro, 29: 538-543. DOI: 10.1016/j.tiv.2015.01.005 